FORMATION Formation emploi

Revue française de sciences sociales

94 | avril-juin 2006

Immigration et marché du travail

Appay B., La dictature du succès, le paradoxe de l'autonomie contrôlée et de la précarisation

L'Harmattan, Paris, 262 p.

Philippe Méhaut

(2) OpenEdition

Journals

Édition électronique

URL : http://journals.openedition.org/formationemploi/2272

DOI : 10.4000/formationemploi.2272

ISSN : 2107-0946

Éditeur

La Documentation française

Édition imprimée

Date de publication : 1 avril 2006

Pagination : 119-122

ISSN : 0759-6340

Référence électronique

Philippe Méhaut, « Appay B., La dictature du succès, le paradoxe de l'autonomie contrôlée et de la précarisation », Formation emploi [En ligne], 94 | avril-juin 2006, mis en ligne le 02 décembre 2009, consulté le 30 octobre 2020. URL : http://journals.openedition.org/formationemploi/2272 ; DOI : https://doi.org/10.4000/formationemploi.2272

(c) Tous droits réservés 


\section{La dictature du succès, le paradoxe de l'autonomie contrôlée et de la précarisation}

Par Philippe Méhaut*

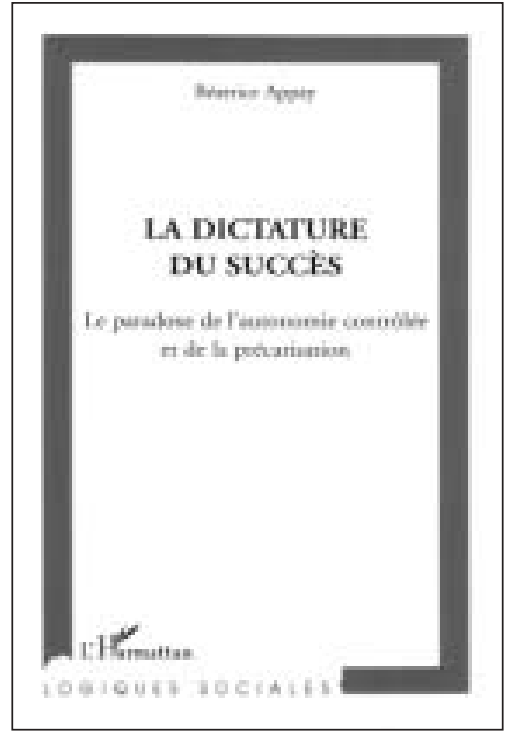

Il y a plusieurs façons d'aborder cet ouvrage : comme une biographie scientifique, comme un apport aux débats autour des nouveaux modèles productifs ou comme une lecture portant à la fois sur la perspective historique et les mécanismes économiques et sociaux en jeu.

Une première lecture peut être faite sur le mode de la biographie scientifique. Issu d'une habilitation à diriger des recherches, l'ouvrage donne à voir, de façon chronologique, le développement d'un ensemble de travaux, qui s'étendent du début des années 80 au début des années 2000. Ils portent aussi bien sur les systèmes de formation que sur différents pans des organisations productives et permettent de construire, d'étendre et d'affiner le concept central de l'ouvrage, celui d'autonomie contrôlée.

Ce concept est défini comme «une dynamique conjointe de concentration et de fragmentation, de centralisation et de décentralisation, d'individualisation et de renforcement d'un pouvoir central..." (p. 241). Il émerge de travaux comparatifs menés par l'auteure sur les systèmes de formation professionnelle français et britannique. Il s'agit, dans les années 80 , de comprendre d'une part le caractère dominé de l'apprentissage en France et d'autre part son passage d'un mode dominant à un mode résiduel, au Royaume-Uni, sous la période Tatcher. Il faut pour cela explorer l'évolution des politiques publiques de formation, mais aussi sortir du système de formation, pour la situer dans les dynamiques organisationnelles des firmes et l'évolution des rapports de travail. Deux hypothèses centrales sont alors posées. La première sur l'évolution des modes de contrôle étatique, la

* Philippe Méhaut est directeur de recherche au CNRS, Lest (Laboratoire d'économie et sociologie du travail, UMR 6126). II participe actuellement à une recherche comparative, menée pour la fondation Russel Sage, sur l'évolution du travail à bas niveau de salaire. Parmi ses publications récentes: "Reforming the training system in France", Industrial Relation Journal, 36: 4, 2005, pp. 303-317; "Competencies based management: what consequences for the labour markets?", Economia et Lavoro, XXXVIII, 1, 2004, pp. 165-180. 
seconde sur les transformations de l'univers de la production.

Celle de l'évolution des modes de contrôle étatique, sur le mode du « vide/rempli » qui, en s'appuyant sur la décentralisation, la crise financière des établissements de formation et le renforcement des injonctions et des contrôles indirects du pouvoir central, va conduire les établissements de formation britanniques à se mouler dans la politique du Youth Training Scheme $^{l}$. Alors que l'observateur attendrait une grande variance dans les politiques d'établissements, celle-ci est paradoxalement plus faible en GrandeBretagne qu'en France, alors même que la décentralisation y est, à l'époque, plus poussée et plus systématique.

Celle ensuite des transformations qui affectent l'univers de la production. Pour saisir la crise, provoquée, de l'apprentissage en Grande-Bretagne, il faut plonger dans l'entreprise. S'y élaborent à cette période les bases d'un nouveau modèle productif: croissance des petites entreprises et, dans le même mouvement, concentration accrue (notamment dans le BTP) ; effondrement des organisations syndicales, des organisations de métier, et, dans le même temps, montée des formes d'organisation du travail apparemment plus autonomes, sollicitant de façon différente l'implication individuelle.

L'autonomie contrôlée serait ainsi une matrice commune aux évolutions des politiques étatiques et à celles des organisations productives. C'est ce qu'il faut vérifier en allant plus loin dans la compréhension des différentes formes de restructurations productives, notamment des différentes formes de sous-traitance, nationales ou internationales, et de leurs conséquences sur les rapports de travail et d'emploi. C'est la deuxième étape de cette biographie scientifique qui voit se réaliser des travaux extensifs, menés en réseau scientifique, sur divers univers de la production (des centrales nucléaires aux hypermarchés). On y valide le concept, on l'affine sur ses deux versants.

Celui du contrôle, avec notamment le rôle des systèmes informatisés centraux et des différentes formes de prescription en cascade sur les sous-traitants ; on est ici sur le registre du contrôle sur et entre

${ }^{1}$ Programme d'aide à l'insertion et à la formation des jeunes développé dans les années 70 . les organisations. Mais ce contrôle sur les organisations est inséparable du contrôle des organisations sur les individus qui les composent : nouvelle organisation des temps de travail (dont l'étude fine du temps partiel), contrôle précis des recrutements pour assurer, par la contrainte monétaire, la flexibilité individuelle. L'exemple finement analysé du recrutement des caissières de supermarché qui ne doivent être dans un milieu familial ni trop pauvre - pour éviter les solidarités - ni trop riche - pour les contraindre à accepter des heures supplémentaires, est particulièrement éclairant.

Celui de l'autonomie, avec les nouvelles formes d'organisation du travail, les espaces de choix. Le recul de la prescription directe, la contrainte de résultat, mais le choix plus ouvert du processus pour atteindre ce résultat ouvrent un espace nouveau au salarié. Le petit entrepreneur qui se met à son compte dispose lui aussi d'un nouvel espace d'autonomie.

Le lien est ainsi fait entre le fil rouge que constitue le concept central de l'ouvrage et ce qu'il permet d'éclairer autour des deux autres notions centrales que sont celles de précarisation et d'individualisation.

Ce premier angle de lecture «biographique» est particulièrement riche. Il permet de comprendre comment, partant d'une intuition dans un champ particulier - celui de la formation - se forge et se développe un concept applicable à d'autres domaines, concept qui vient alors éclairer diverses facettes de la transformation des rapports sociaux.

Un deuxième mode de lecture est celui de l'apport de l'ouvrage aux multiples débats autour des nouveaux modèles productifs et de leurs conséquences économiques et sociales.

L'autonomie contrôlée est ici mise en miroir aux différentes approches de la coopération forcée, de l'implication contrainte, ou de la double régulation de J.-D. Reynaud (1989). Ce concept se veut à la fois dialectique (c'est la tension entre ses deux dimensions qui importe, même si le deuxième terme - le contrôle est parfois plus exploré que le premier) et généraliste : clé de compréhension globale du monde du travail, mais aussi plus généralement des évolutions à l'œuvre dans le champ des politiques publiques ou d'autres univers. On est en effet tenté par la transposition par exemple au champ du workfare, avec les contrôles renforcés sur les bénéficiaires d'allocation et les injonctions à la reprise d'une activité, gage d'auto- 
nomie, ou encore dans le champ de la santé avec le libre choix du médecin référent qu'accompagne là encore un contrôle centralisé plus strict de la pratique médicale et de la consommation de soins.

L'éclairage apporté sur le processus de précarisation sociale mérite lui aussi que l'on s'y attarde. Un schéma (p. 132) résume bien la pensée de l'auteure. La précarisation économique (sous sa double dimension de précarisation des structures productives et de précarisation salariale (chômage, statut d'emploi...)) se conjugue avec la précarisation de la protection sociale (droit du travail, situations hors travail). La santé au travail est alors un révélateur de ce double processus. Plusieurs chapitres fournissent des analyses empiriques fouillées de la mise au chômage, du temps partiel, des figures sexuées de cette précarisation qui touche, sous des formes diverses, aussi bien la caissière que le petit cadre de supermarché. L'ouverture sur les questions de santé au travail (et ses nouvelles figures, stress, harcèlement...) est importante, même si rapidement traitée, car elle rejoint les constats statistiques sur la dégradation conjointe des conditions de travail et de certains indicateurs de santé. Une des clés d'interprétation résulterait alors dans l'injonction paradoxale que contient l'autonomie contrôlée et qui pèse fortement sur la subjectivité des salariés. Là encore, en élargissant le propos, on pourrait trouver des éléments d'explication du «paradoxe de la subjectivité » : alors que les indicateurs « objectifs » de la stabilité de l'emploi ne montrent pas une instabilisation généralisée, le sentiment de précarité est très fort; ou encore, alors que certains indicateurs objectifs des conditions de travail (recul des accidents du travail, baisse du temps de travail) semblent traduire une amélioration, les enquêtes auprès des salariés sur les conditions de travail, sur la santé au travail expriment plutôt une dégradation.

Il reste une troisième ligne de lecture, plus critique celle-là, qui porte sur la perspective historique, les mécanismes économiques et sociaux convoqués pour l'explication et sur les contre-tendances.

Bien que se posant en position critique dans la controverse des «post» industriels et «anti-post», l'ouvrage, par sa forte insistance sur toutes les dimensions négatives liées à la précarisation, n'échappe pas à la même critique. On a la curieuse impression qu'il y a eu un âge d'or (celui des Trente glorieuses, un âge plus ancien?) qui sert de référence implicite et de grille de lecture aux évolutions à l'œuvre. Pour revenir aux questions de formation, l'apprentissage à la britannique, certes dé-construit dans la violence, était-il vraiment l'âge d'or? Et que dire des évolutions importantes du système éducatif et de formation de ce pays dans les dix dernières années ? Du côté français, peut-on vraiment voir dans la décentralisation une figure noire qui s'opposerait à une centralisation bénéfique et hélas révolue? Les mêmes questions pourraient être posées à propos du temps partiel et du travail féminin. L'essor irréversible du taux d'activité féminin, l'entrée massive des femmes dans certaines activités ne sont vus qu'au prisme du temps partiel contraint et de ses méfaits réels. Mais comment expliquer le maintien - au moins dans la période actuelle - d'un taux de fécondité largement supérieur à celui de la plupart des pays européens si la précarisation de la protection sociale est si avancée et si unilatérale?

Si l'on voit bien, même si ce ne sont pas les termes de l'auteure, comment les processus de globalisation, de financiarisation contribuent à refermer le piège de la «dictature » du succès, on a parfois l'impression d'une forme de complot du capital et du politique. D'autres acteurs sont très largement absents, ne serait-ce que le consommateur qui n'est pas totalement étranger, par ses comportements certes eux aussi modelés, aux transformations du modèle productif. Il reste alors au moins deux inconnues. La première est la portée «universelle» de l'analyse proposée. Certains pays semblent mieux résister que d'autres aux conséquences de cette dictature, alors même qu'ils sont parfois plus soumis que la France aux contraintes mondiales. De même, certains groupes de salariés semblent "gagnants » alors que d'autres sont perdants (voir par exemple les travaux de Paugam (2000) ou ceux de Baudelot et Gollac (2003)). La seconde est son caractère irréversible. Comment situer dans ce cadre certaines tendances à la re-régulation qui émergent au Royaume-Uni, ou encore l'émergence (certes fragile et incertaine) de contre-pouvoirs qui n'empruntent pas nécessairement la voie classique de la lutte syndicale?

Mais tel n'est pas le propos de ce livre, qui revendique haut et fort sa posture : renouveler les fondements de la critique sociale qui aurait manqué le virage des nouveaux modèles productif. À ce titre, dès lors que l'on accepte cette posture, sa vigueur est décapante et a le mérite de contribuer au débat. 


\section{Référence de l'ouvrage}

Appay B. (2005), La dictature du succès, Le paradoxe de l'autonomie contrôlée et de la précarisation, L'Harmattan, Paris, 262 p.

\section{Bibliographie}

Baudelot C., Gollac M. (2003), Travailler pour être heureux? Le bonheur et le travail en France, Paris, Fayard.

Paugam S. (2000), Le salarié de la précarité, Paris, PUF.
Reynaud J.-D. (1989), Les règles du jeu, l'action collective et la régulation sociale, Paris, Armand Colin. 